\title{
Network Lifetime Optimization in Sensor Cloud
}

\author{
Prashant Sangulagi \\ Department of ECE, BKIT Bhalki-585328, and affiliated to \\ Visvesvaraya Technological University, Belagavi, Karnataka, INDIA \\ Email: psangulgi@gmail.com \\ Ashok V. Sutagundar \\ Department of ECE, BEC Bagalkot -58710, INDIA \\ Email: sutagundar@gmail.com \\ Tabrunisa Abdul Rashid \\ Department of ECE, BKIT Bhalki-585328, INDIA \\ Email: tabrunisa786@gmail.com
}

\begin{abstract}
Sensor cloud is a new technological development and improvement of numerous day to day life applications. It is in the form to embed for cloud computing with wireless Sensor network. As wireless no more furnishes with processing, information storage and exploring but it all done by the use of cloud and also it help to overcome problems like efficiency, lifespan and energy supply. The proposed methodology works on push pull mechanisms to save the node's energy and to improve the network lifetime. The two way communication from sensor to cloud is made possible along with making nodes in active and sleep mode to preserve the node's energy and prolong the lifespan of network. Push mechanism is responsible for sending the active nodes data to cloud server and push is operated for two ways communication i.e. user request are executed by communicating with sensor nodes from cloud server and intern sensor network provide the required data to cloud server. The result shows that there is an improvement in saving node energy, less end to end delay and hence prolonging network lifetime.
\end{abstract}

Keywords - Wireless Sensor Networks, Sensor Cloud, Optimization, Network Lifetime.

\section{INTRODUCTION}

\subsection{Wireless Sensor Networks}

$\mathrm{W}$ that reason useful element for knowing the imaginative and prescient of the Internet of Thing (IoT). They are composed of wide variety of small size sensor and WSNs sustainably utilized in many administrations domains among them in fitness check, health-care, commercial sensor networks, military, environmental tracking, domestic automation, habitat tracking, agriculture and manufacturing and so on. To conquer WSNs shortcomings, and permit an extra computing and storage competencies, the cloud becomes proposed and the sensor nodes with low capabilities with regard to reckoning storage and control. Indeed, a cloud offers scalable high performance computing and large garage infrastructure for data storing and actual-time processing as in [1].

The sensors are placed within target proximity. In sensing community, the main hassle is community lifetime (network lifetime). The cost and size of network limits the on hand electricity within sensor network to feel the bodily phenomenon. A standard method to energy saving is to apply mechanisms for low-priced power management. This method is applied on programming the sensing activity. From [2] and so, accomplice degree operation mode alternates energetic or reveal inactive battery states to extend the community duration. Some of challenges in WSN are addressed as real time, in power division management, Network Scale and Time-Varying
Characteristics of WSN and Management at a Distance [5] and there five varieties of WSNs namely, Terrestrial WSN, Underground WSN, Underwater WSN, Multi-media WSN, Mobile WSN [3] [4]. Issues in WSN are classified as Design Issues and topology issue $[5,6]$. The purpose for WSNs are Environmental tracking, Automobile detection, Greenhouse monitoring, Windrow Composting, Flare Stack monitoring, Medical field, Industrial purpose, Military Application [7] [8].

\subsection{Cloud Computing}

$\mathrm{Be}$ fond of cloud computing is most modern term for protracted desired vision of computing expediencies, it grant a ambiance in support of basis allotment here requisites of preeminence edifices, middle-ware and relevance improvement proposal, and dealing relevance [9]. Cloud Computing is form for permit everywhere, wellsituated happening claim way in to a mutual team of be patterned computing possessions to facilitate can be in haste victual and on the loose with nominal supervision exertion before examine source relations. It is combination for various anterior tools that have qualified next to special charge and here diverse situation. The cloud form market is increasing significantly. The analysis also brings into being to facilitate $41 \%$ of for profit unit run momentous tasks on public cloud. But growth of cloud has brought some security challenges. These security issues should be identified and analyzed here categorize to compose cloud services more secured with consistent. The characteristics of cloud computing are like computing competence, trustworthy, continuation and provision of computer services [10]. Cloud end user has business enterprise that 
keeps up a commercial enterprise affiliation in the midst of, and applies offerings on or after Cloud Company. Cloud giver has in anyone otherwise agency for growing provider on hand to engrossed celebration. Cloud assessor has revelry to could perform sovereign assessment of cloud organization, overall presentation, statistics agenda operation also safety of confuse utilization. Cloud negotiator has a unit to facilitate deals with usage, recital along with release of cloud uses and agent associations among cloud purchasers and companies. Cloud service has path so as to gives set-up and delivery attributed to cloud offerings since cloud carriers toward clients. Cloud Computing has service models serving as IAAS, PAAS and SAAS [9] [11]. It's still challenging in cloud computing to provide better security, integrity, reliability, availability along with hardware and software compatibility [11].

\subsection{Sensor Cloud}

The idea of sensor cloud as regards to accruing the sensor information from one to another sensor location and also it related with sensor virtualization. Sensor cloud [1] is greater form of cloud computing to direct spread of sensor in the network area and it enable to collect more amount of data as it has more amount storing capacity and processing ability with the help of WSNs and cloud sink, this happen when we integrating cloud computing and WSNs. The sink node acquires more amount of information from various senor nodes and passes to cloud to save the information of sensor. The data can be used by user by cloud via internet and it will take help of algorithm. Sensor cloud can be illustrated by the help of three tiers architecture [1, 32] where it is divided hooked on three layers as shown from figure 1 .

Sensor Layer: It holds many greater numbers of WSNs and each of those networks has wide variety of sensors which help in managing the temperature, pressure and moisture. The sensor device user can be registered or it can be deleted and it has limited amount of energy supply to sensor because they are managed by battery. In sensor layer the information can be transmitted by hop by hop mechanism where it is arranged in circular group in our paper and many routing protocols are been used for transmitting such information to sensors because to have communication between the sensors.

Sinks Layer : It sedate of lots of sink nodes that are liable for gathering information from each and every sensor which are present in the sensor network as sink will save information periodically send to the cloud layer by same transmission rate. Sink layer is responsible for mapmaking of sensor between virtual and physical sensors in cloud as it knew that source and destination node.

Cloud Layer: It also sedate of one or several that host and control the applications of end user, it can be achieved on virtual machines in cloud. Consumers demands for virtual sensors through cloud implementation in order to handle physical environment. The applications of sensor cloud include disaster and environment monitoring, health care, agriculture field, telemetric, and many more [12]. The sensor cloud enables accelerated information storage, processing strength, visualization, scalability, data analysis with availability of resources anywhere and anytime [12, 13]. Some of the major issues of sensor cloud are [14], bandwidth utilization, better connection, optimization, security etc.

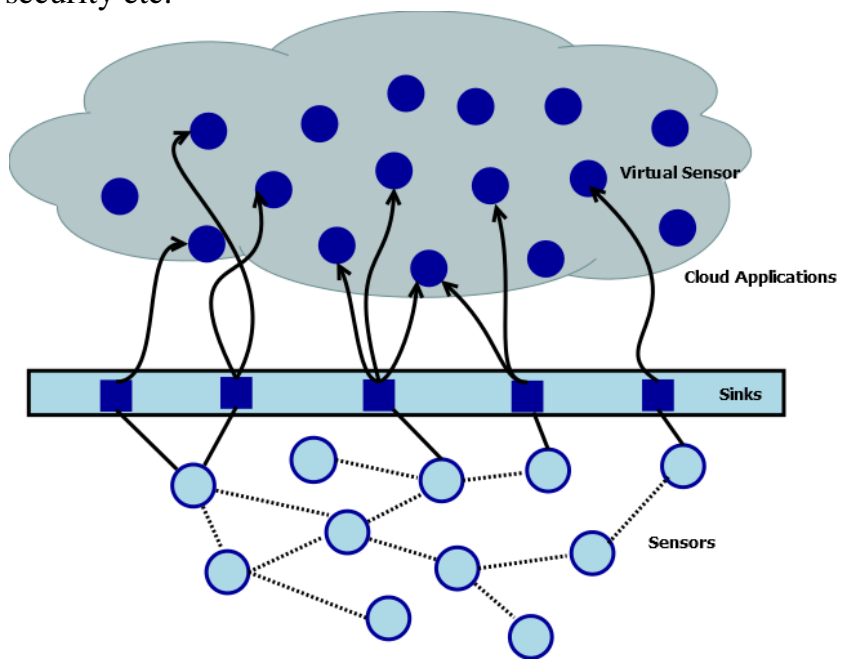

Fig. 1: Layers of sensor cloud

In this paper we are going to deal with an optimization trouble that increases the life of sensor cloud schemes. A push-pull mechanism is used to improve the overall network lifetime by utilizing the sensors in optimal way. Approaching every node is done in push mechanism and the whole of packets dispatched is same as the full of acquired packets then information packets generated at sensor node that is toward sink node. The power used by push mechanism with the aid of the storage battery powered sink is identical to power expended in acceptance and broadcast of incoming sensor information. The power has dissipated in Pull mechanism by way of the battery powered sink okay is equal to power ate up in reception and transmission of demands obtained from the cloud and reception and transmission of sensor information.

The paper is organized as follows; section 2 gives some of the previous work done on WSN, Cloud Computing and Sensor Cloud. Section 3 describes the proposed work. Section 4 provides simulation of work of the proposed scheme. Section 5 discusses 5 the result and discussion. And finally section 6 concludes the paper.

\section{LITERATURE SURVEY}

Literature survey consented out that for basic increasing the energy efficiency of sink node along with sensor to achieve maximize lifespan of sensor cloud, where it has issue of limited or short lifespan of sensor cloud which can be achieved by using the protocols and mechanisms such as push and pull mechanism and shortest path protocol.

J. Carter et al. [15] set up the virtual machine requirements for assorted processing, input output processing and 
memory where the requirements are increases by received time and the transmitted time due to power action in the heuristic by increasing interval time which can be launched by the help of virtual machine and simulation can be achieved by saving its energy of performance up to $55 \%$ and there is limitless in virtual machine duration is achieved.

P.V. Ramakrishna et al. [16] find out the genetic algorithm for the conservation of energy in the system for better communications and measurement in the system. Where it provide information about the result of DSM consignment transmission of service application in the helpful and valuable area for decreasing the power consumption automatically by using algorithm, also the power get conserved at user side and therefore efficiency of such communication will increases.

S. Avallone et al. [17] studied the efficient energy routing by using stabilizer constraints. In this the routing technique is used as online or that is said by using internet services to maximize the bandwidth usages and decreasing the power intake of the system which has been used. The technique used is E2-MCRA which searches the appropriate route for the source and destination for communication between the node points.

Mallanagouda Patil et al. [18] described that the routing protocol is classified in seven ways to provide efficient and optimum routing path for sensors which are classified on bases of routing parameters which use to communicate between sensors and to have achieve the stability, scalability again efficiency and reduce the power consumption these all thing are covered in detail in the that survey.

Y.Li et al. [19] have discussed the WSNs schemes for the quantitative measurement and source location designs. In this source location scheme (SLP) scheme is used for the privacy protection of information where there is secured data has to transfer for source to destination without any interruptions. As the system build at low cost and by using less power devices, in WSNs the routing scheme is used is well efficient for communication between sensor nodes and it is well suited for practical application of day today life.

Apurva, Pusatkar et al. [20] have described WSNs implementation for the real time system monitoring in agriculture field application. In the application of agriculture many parameters are considered such as soil moisture, light, temperature and also humidity other thing are carefully observed for controlling the changes by using the sensor monitoring devices where hardware components are used to senses the changes of data. Here they have used the TCP/IP protocols and microcontrollers for detecting the variation in the system, this setup has improved with low cost and many sensors are used to monitor the process and controlling the work flow in agriculture.
Haider et al. [21] described energy efficient application oriented sensing system for monitoring the agriculture field. Many key parameters are considered in the field for control processing such as disease controlling in plants, weed control and reducing pest control. It has many sensor, microcontroller unit and Radio Frequency module are used in the system for achieving the efficient system and wireless technology and protocols are used such as Bluetooth, Wi-Fi, ZigBee, GPRS/3G/4G and SigFox.

Sayantani Saha et al. [22] proposed the work on data management by secure way of sensor cloud by integrating with surrounding environment by using protocols and routing techniques to achieve high speed communication in secure way and this is best when the there is more traffic in the network environment in one of such practical application it can be used.

Y.Li et al. [23] discussed on WSNs privacy and authentication. Where hop by hop authentication techniques is used for send privacy data from the source node this work is mainly used in security purpose application and elliptic curve cryptography scheme is used in this.

San Diego et al. [24] have worked on SLP of WSNs by dynamic routing protocol where it provide the privacy for source location to have secure data transmission to the destination with highly efficient, low latency and less energy consumption by using best random routing protocol techniques.

\section{PROPOSED WORK}

In sensor networks, optimization is done separately in every layer, but in sensor cloud the same strategies are not perfectly fit to do the mechanism so after broadly study in $[25,26,33]$ optimization technique for push and pull mechanism is used. To improve the performance in sensor cloud, there is optimization problem because of this reason we are seeking help of push and pull communication mechanism to improve overall efficiency, network lifetime and to overcome sensor cloud problems which is related to our work [27, 28].

\subsection{System Environment}

In [29] a proposed condition there is three tier structural designs known as cloud, edge and beneath. It strengthens the scalability and there will be an energy efficiency increment by means of two optimizing algorithms which broadcasts information between cloud, sensor layer and edge layer. The energy using up of sensor nodes is with respect near the performance optimization process. Push and pull mechanism which communicates between cloud and sensor layer during edge layer. There is a proposed condition of optimization algorithm of numerous features with respect to sink node and sensor node. The energy consumption, bandwidth and information provider is with respect to performance of optimization. This paper is distinct from present work which is centered on rate of information transmission. This work frames problems on 
optimization which accelerates energy curtailment of sensor and sink layers with respect to sensor cloud lifespan.

In sensor cloud, the way of gathering information from sensors has been classified in to two mechanisms which are push and pull in push method, the combined records will be sent to sink layer time to time by the sensor. In pull method, there is a process of gathering the data and sensor statistics which is plead of circular ride. In order to be saved in cloud the data has to be send cloud and it also offers the large device capacity and capabilities for processing the information. By the link of WSN and cloud through sink node the data can be allow to gather in the sensor cloud. User can fetch the data through Internet by the use of cloud. The systematic presentation of layers and method has been shown in figure 2 .

\subsection{Implementation and Working}

Modules: the modules which has used in our paper are as follows

\section{Shortest path routing algorithm}

The routing algorithm used in our paper is shortest path routing protocol [1]. From study [29] it can be defined as a shortest path search a root containing less rate between two peaks of sensor nodes and the main goal of this protocol is to achieve its less time delays between the nodes. The sensors has inner and outer circle where inner circle make exploit of the shortest conduit near pass information to sink node by using two steps that are the sensor use round routing around the network and find he short root of the sink node and after the confirmation of path the data has been send to sink node. This make the reduction of distance sink node and sensor nodes by this, it will achieve the protocol routing.
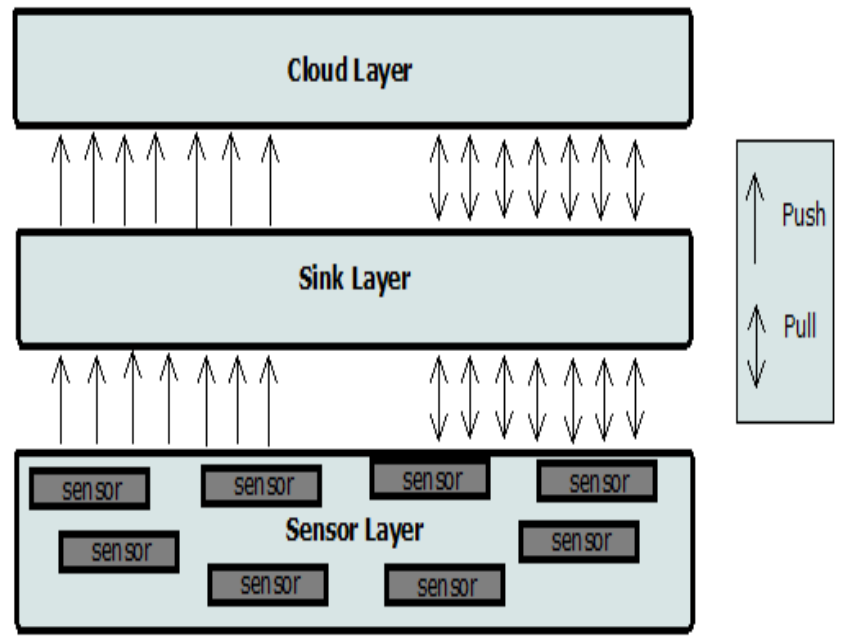

Fig. 2: Push and Pull method in Sensor-Cloud system

\section{Node creation}

The multiple node formation can be designed on the bases of three dimensional theory where it $\mathrm{z}$ axis provide the approximate 0.33 value and the node is to transmit the information around the flat ground which may take value zero. Because of this the three axes $\mathrm{X}, \mathrm{Y}$, and $\mathrm{Z}$ are adjusted to action of node. NS2 is used to implement wireless sensor network and while NS2 create node it will check the energy constraint in the sensor node and we are using sensor to be use less energy for transmission of packets to sink node. In our paper we have created 63 nodes in the form of cluster where it has one sink node 0 and other 62 are source nodes. The node will define when they are transmitting packets as consuming energy mode while other node will be in state of saving energy.

\section{Push and Pull Mechanism}

We are using push and pull method in our paper to achieve the optimization in sensor cloud and where it increases the lifespan of sensor cloud networks. the mechanism is shown figure 2 where sensor layer send the gathered data to the sink layer this is done by push method and pull provide us data on accepting request. The sensor node are in active state while transmitting data which show as consuming energy and other while receiving the data packets they also become in active state apart of those node other node are in inactive state that is energy saving state.

\section{SIMULATION AND RESULT}

In this section we are discussing the simulation parameters used for the execution of proposed work along with the obtained results. The proposed work is simulated using the NS-2. Network simulator (NS-2) [31] is an object oriented open source simulation tool use to get accurate result. The basic view of NS-2 is as shown in figure 3. It is inscribed in OTCL (object oriented variant of TCL) and $\mathrm{C}++$ to implement route information and manage.

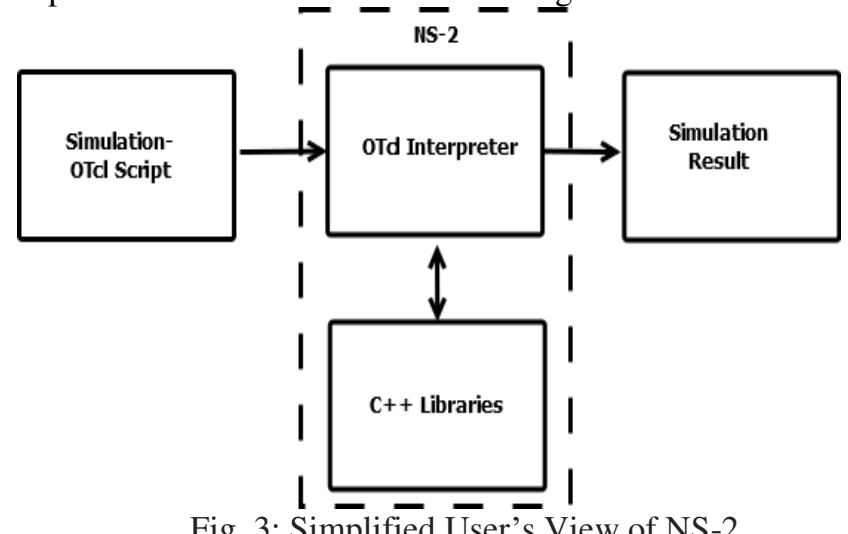

4.1 Results

Once the sensor nodes are created by the system, it will ask for the how many packets to be transmitted to the system, then it we can give up to 60 packet for the transmission as we have design the system and the we have to choose the source node from 0 to 62 and destination is fixed i.e sink node. The shortest path is created by using shortest path algorithm. The arrangement of sensor node in concentric circular manner as shown in figure 4 , here in this paper we have created 63 nodes and one sink destination node. The arrangement of node in network animator and the file is called as out. Nam and here sink node at the center of all sensors. In figure there is timing scale at below where it 
provides transmission timing in milliseconds and we can also increase the speed of transmission of packets.

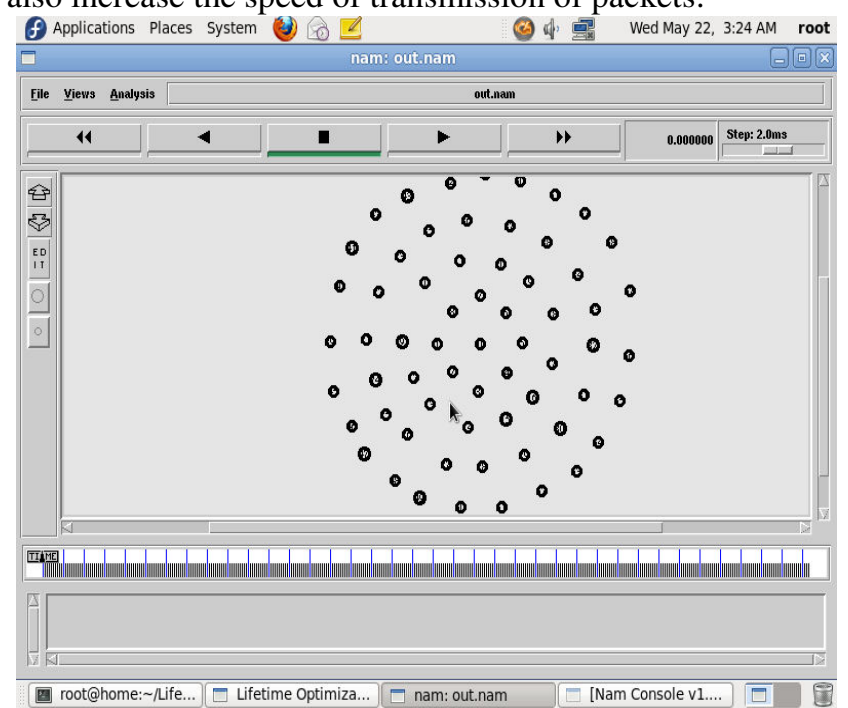

Fig. 4: Network animator starting phase

The packet will send from source node such as we have given 44 where we are transferring 40 packets to the sink node, here in this paper we are considering sink or destination node as node 0 . At the starting phase of transmission all nodes are in energy saving mode, when source node is ready to send packet to routing path created by using the algorithm.

The all sensor node are showing its value of GPI (Genuine progress indicator) which is equal for all that is one, then the source node send the RTS (Request to send) message to other neighbor nodes for sending packets is that path is free or busy, if the path clear then node will send the CTS (clear to send) massage to requested node as shown in figure 5 .

Then this process continues till the sink node and lastly they will attain QPI (quick path interconnection) to send packet and set routing path. The all nodes at first stage they will at saving energy state and after while they sharing packets they are consuming energy state as shown in figure 5.

\subsection{Performance Parameter}

\section{Packet delivery ratio}

Figure 6 shows the graph of packet delivery ratio. It's a total number of packets generated by the nodes to the number of packets saved into the cloud server. From the graph it is clear that the proposed methodology is doing better compared to improved leach and PEGASIS. LEACH and PEGASIS have only basic algorithm to minimize some amount of packets which are redundant in nature, but our proposed work checks all the data and on regular basis redundant data are discarded by comparing with the threshold.

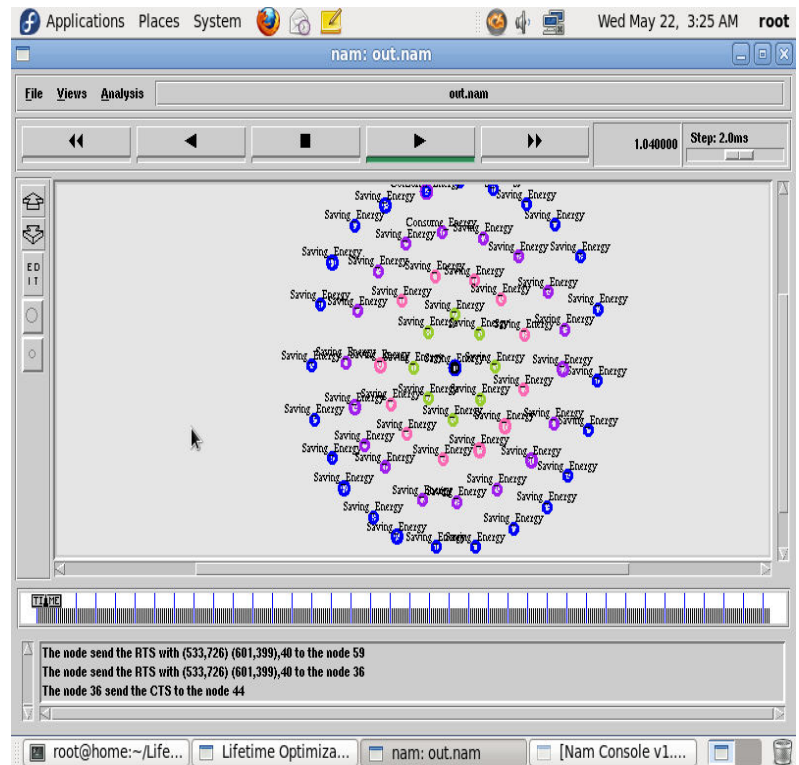

Fig. 5: Nodes in energy saving and consuming mode

\section{End to end delay}

End to end delay is used to check the total time consumed by source node to send its packets to destination node. End to end delay graph is presented in figure 7 . In this the time taken by the proposed work, I-LEACH and PEGASIS to reach destination from source node are checked. Due to the fast response push-pull algorithm the time taken by the proposed work (in milliseconds) is very less compared to ILEACH and PEGASIS. Fast delivery is possible from the proposed work.

\section{Energy Consumption}

Energy consumption is the total energy consumed by the system to complete one full task. Lots of node energy is consumed due to transmission and reception of data. The result shows that our proposed work consumes very less energy and prolongs the network lifetime with great extent than compare to both conventional methods.

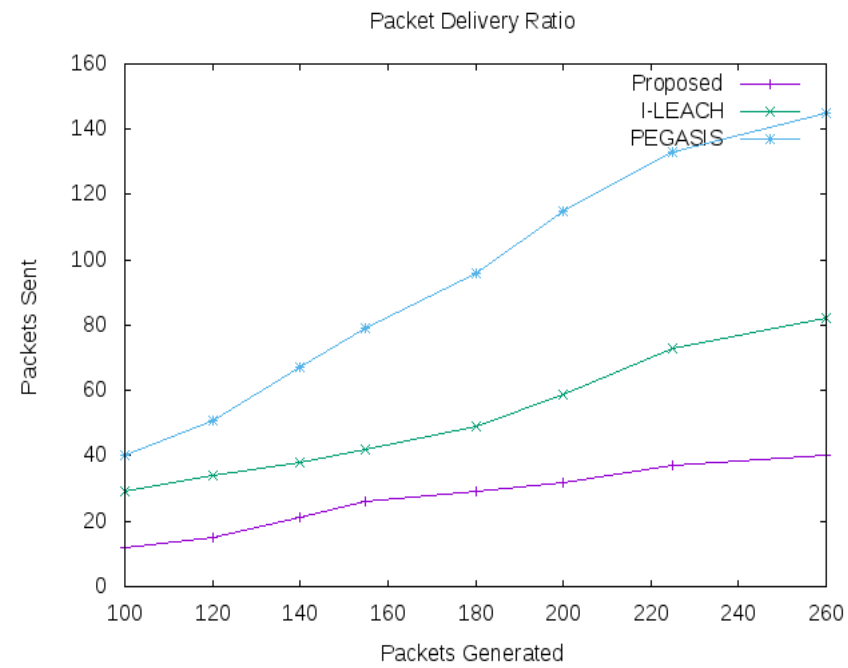

Fig. 6: Packet Delivery Ratio 


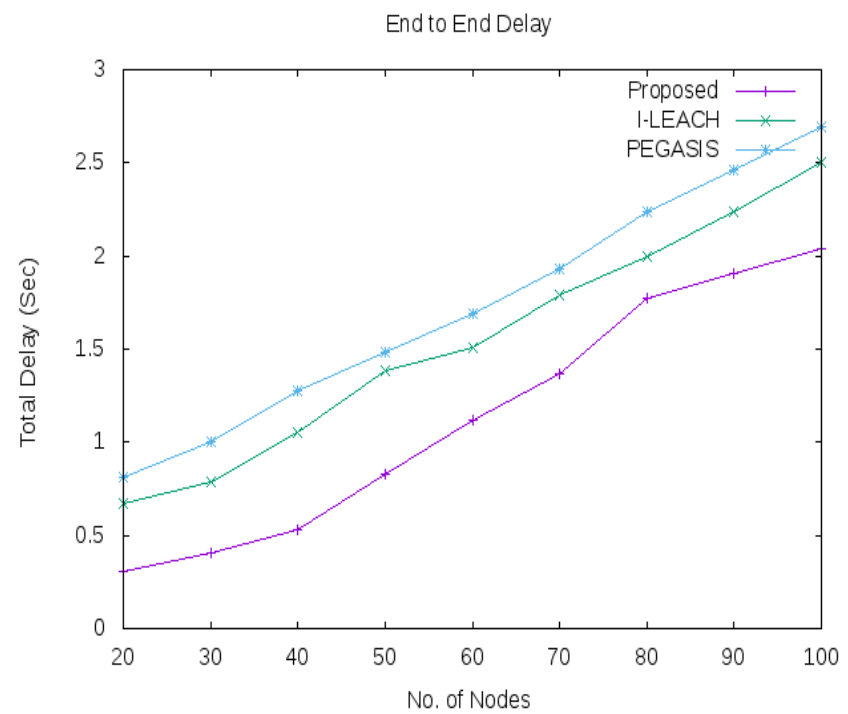

Fig. 7: End to End Delay.

\section{CONCLUSION}

A WSN plays a vital role in sensor cloud to assemble and process the information from various networks. We are solving the problem of sensor cloud where it has the performance issue and less lifespan which has been solved to some extent, as we are increasing the strength by saving sensor energy at the consumption time and an ideal state, hence increasing the life span and optimizing the sensor cloud. The issue is uttered by increasing the lifespan of sensors on the basis of push and pull mechanisms and protocols for making quick connectivity between senor nodes which make ease for transfer packets and communication between source and sink node. As from simulation point of view the sensors are consuming less power at the time of mechanism which has been optimized. The lifespan of sensor network is enhanced about $10 \%$ to its efficiency.

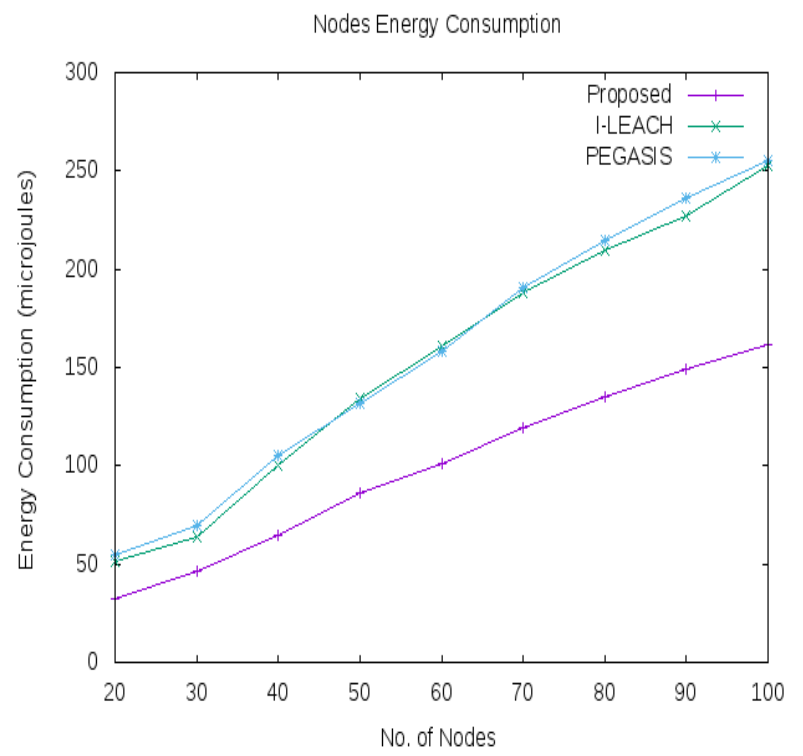

Fig. 8: Total Energy Consumption

\section{ACKNOWLEDGEMENT:}

The authors thank AICTE for the support and the college for doing the work. The work is funded by AICTE grant for carrying out the project "Resource Management in Internet of Things" Ref. No. File No. 840/RIFD/RPS/POLICY1/2016-17 dated August 02, 2017.

\section{REFERENCES}

[1] Leila Ben Saad, Bernard Tourancheau, Lifetime Optimization of Sensor-Cloud Systems, IEEE, 2015 7th International Conference on New Technologies, Mobility and Security (NTMS), France, 2015.

[2] Mukhdeep Singh Manshahia, Wireless Sensor Networks: A Survey, International Journal of Scientific \& Engineering Research, 7(4), 2016, 710-716.

[3] Hina Tandel, Prof. Rakesh Shah, A Survey Paper on Wireless Sensor Network, IJSRD - International Journal for Scientific Research \& Development, 5(10), 2017. 907909.

[4] Dr. N. Hariharan, Sreelekshmi, A Survey on Wireless Sensor Networks, International Journal of Scientific Engineering and Science, 1(11), 2017.

[5] Indu, Sunita Dixit, Wireless Sensor Networks: Issues \& Challenges, IJCSMC, 3(6), 2014, 681-685.

[6]. Gowrishankar.S, T.G.Basavaraju, Manjaiah D.H, Subir Kumar Sarkar, Issues in Wireless Sensor Networks, Proceedings of the World Congress on Engineering WCE 2008, 1, 2008.

[7]. Monika Nanda, Upendra Kumar Singh, Bhimtal, Nainital, Haldwani, Nainital, A Survey on Wireless Sensor Network Technologies, Recent Advances and Applications, international research journal of engineering and technology (IRJET), 3(7), 2016.

[8] Himani Chawla, Some issues and challenges of Wireless Sensor Networks, International Journal of Advanced Research in Computer Science and Software Engineering Research Paper, 4(7), 2014, 236-239.

[9] Palvinder Singh Er. Anurag, Survey Paper on Cloud Computing, Jain International Journal of Innovations in Engineering and Technology (IJIET), 3(4), 2014, 84-89.

[10] Md. Sakib Bin Alam, Cloud Computing Architecture, Platform and Security Issues: A Survey, World Scientific News 86(3), 2017.

[11] Harshitha. K. Raj, A Survey on Cloud Computing, International Journal of Advanced Research in Computer Science and Software Engineering Research Paper, 4(7), 2014.

[12] Atif Alamri, Wasai Shadab Ansari, Mohammad Mehedi Hassan, M. Shamim Hossain, Abdulhameed 
Alelaiwi, and M. Anwar Hossain, A Survey on SensorCloud: Architecture, Applications, and Approaches, International Journal of Distributed Sensor Networks, 2013, 1-18.

[13] http://www.ntu.edu.sg/intellisys.

[14].Wasai Shadab Ansari, Atif M. Alamri, Mohammad Mehedi Hassan, and Mohammad Shoaib, A Survey on Sensor-Cloud: Architecture, Applications and Approaches, College of Computer and Information Sciences King Saud University, Riyadh, KSA, 2013.

[15]. J. Carter and K. Rajamani, Designing EnergyEfficient Servers and Data Center, Computer, 43(7) 2010, 76-78.

[16] V. Siddhartha, P.V. Ramakrishna, T. Geetha and A. Sivasubrama- niam, Automatic generation of energy conservation measures in buildings using genetic algorithms Elsevier Energy and Building, 2011, 2718-2726.

[17] S. Avallone, G. Ventre, Energy efficient online routing of flows with additive constraints, 56(10), 2012, 23682382 .

[18] Mallanagouda Patil, Rajashekhar C. Biradar, A Survey on Routing Protocols in Wireless Sensor Networks, $18^{\text {th }}$ IEEE International Conference on Networks(ICON), 2012, 86-91.

[19] Y. Li, J. Ren, and J. Wu, Quantitative measurement and design of source-location privacy schemes for wireless sensor networks, IEEE Transactions on Parallel and Distributed Systems, 23(7), 2016, 1302-1311.

[20] Apurva C. Pusatkar, Vijay S. Gulhane, Implementation of wireless sensor network for real time monitoring of agriculture, International research journal of engineering and technology, (IRJET), 3(5), 2016, 9971003.

[21] Haider Mahmood Jawad, Rosdiadee Nordin, Sadik Kamel Gharghan, Aqeel Mahmood Jawad and Mahamod Ismail, Energy-Efficient Wireless Sensor Networks for Precision Agriculture: A Review, Sensors, 17(8), 2017.

[22] Sayantani Saha, Secure Sensor Data Management Model in a Sensor - Cloud Integration Environment, 2015 Applications and Innovations in Mobile Computing (AIMoC), Kolkata, 2015.

[23] Y. Li, J. Li, J. Ren, and J. Wu, Providing hop-by-hop authentication and source privacy in wireless sensor networks, In IEEE INFOCOM 2012, March 25-30, 2012.

[24] San Diego, Source-location privacy through dynamic routing in wireless sensor networks, In Proceedings of IEEE INFOCOM 2010, March 15-19, 2010.
[25] X. Liu, Q. Huang, and Y. Zhang, Balancing push and pull for efficient information discovery in large-scale sensor networks, IEEE Transactions on Mobile Computing, 6(3), 2017, 241-251.

[26] T. Zi-Jin, G. Zheng-hu, O. Zhen-Zheng, and X. Jin-Yi, Two new push pull balanced data dissemination algorithms for any-type queries in large-scale wireless sensor networks, In International Symposium on Parallel Architectures, Algorithms, and Networks, 2008, 111-117.

[27] Y. Xu, S. Helal, M. Thai, and M. Scmalz, Optimizing push/pull envelopes for energy-efficient cloud-sensor systems, In Proceedings of the $14^{\text {th }}$ ACM International Conference on Modeling, Analysis and Simulation of Wireless and Mobile Systems, 2011, 17-26.

[28] D. H. Phan, J. Suzuki, S. Omura, and K. Oba, Toward sensor-cloud integration as a service: Optimizing three-tier communication in cloud integrated sensor networks, In Proceedings of the $8^{\text {th }}$ International Conference on Body Area Networks, 2013, 355-362.

[29] Amgad Madkour, Walid G. Aref, Faizan Ur Rehman, Saleh Basalamah, Mohamed Abdul Rahman, A Survey of Shortest-Path Algorithms, Data Structures and Algorithms, 2017, 1-26.

[30] Genita Gautam, Biswaraj Sen, Design and Simulation of Wireless Sensor Network in NS2, International Journal of Computer Applications, 113(16), 2015, 14-16.

[31] Venkatatamangarao, Dr. M. Raghavender Sharma, Dr. K. R. Balaji, Network Simulator Version 2 for VANET, International Journal of Scientific Research in Computer Science, IJSRCSEIT, 2(5), 2017, 878-887.

[32] Prashant Sangulagi, Ashok V Sutagundar, Stelvarani $\mathrm{S}$, Storage of Mobile Sensor Data in Clouds using Information Classification Algorithms, Int. J. Advanced Networking and Applications, 10(3), 2018, 3893-3897.

[33]. Shivshanker. P. Biradar, Dr. T.S. Vishwanath, Network Lifetime Maximization of Sensor Network Based on Energy Aware Source Tree Routing, Int. J. Advanced Networking and Applications, 10(2), 2018, 3788-3793. 